\title{
Efecto de la asesoría dietética sobre la frecuencia de consumo de azúcares en niños.
}

\author{
Effect ofdietary counselingon the frequency ofsugar consumptionin children.
}

Rita Orihuela-Campos ${ }^{1, a}$, Abraham Meneses-López ${ }^{2, b}$, Carlos Heredia-Azerrad (†) ${ }^{3, c}$.

\section{RESUMEN}

Objetivo: Determinar si la asesoría dietética tiene algún efecto sobre la frecuencia diaria de consumo de azúcares extrínsecos (FCAE) en pacientes de 3 a 13 años de edad atendidos en la Clínica Estomatológica Central de la Universidad Peruana Cayetano Heredia. Materiales y métodos: La población muestral estuvo conformada por 2865 historias clínicas. Los datos se ordenaron y procesaron mediante estadística descriptiva. Se usó la media aritmética y la desviación estándar de la FCAE. Se elaboraron gráficos de líneas para reflejar las tendencias a través del tiempo y los grupos fueron comparados a través de la observación directa de las diferencias. Resultados: Se encontró que los niños de mayor edad reciben menor número de asesorías dietéticas. Los niños mayores tienen una mayor FCAE promedio que los menores y que la FCAE promedio es menor cuando el paciente recibe más de una asesoría dietética.

Palabras Claves: SACAROSA, DIETA,USO TERAPÉUTICO DIETOTERAPIA.

\section{ABSTRACT}

Objective: To determine the effect of dietetic assessment on the intake frequency of extrinsic sugars (IFES) in 3- to 13-yearsold-patients attended in the School of Dentistry from Cayetano Heredia University in Perú. Materials and Methods: The study group included 2865 clinical reports. All the information was ordered and processed by descriptive statistic. Mean and standard deviation of IFES were used. Also, line graphics were made to show the trends through the time. The groups were compared using straight analysis of differences. Results: It was found that elder children receive less dietetic assessments and their IFES is higher than younger ones. Additionally, it was demonstrated that patients who had received more than one dietetic assessment decreased their mean IFES.

Key Words: SUCROSE, DIET, THERAPEUTIC USE, DIETTHERAPY

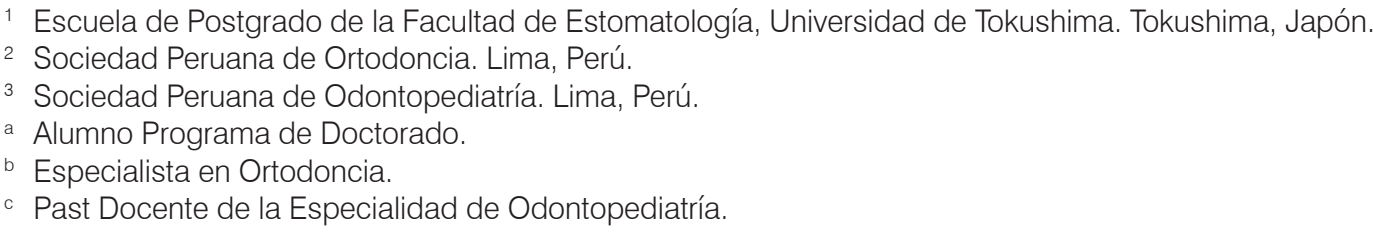




\section{INTRODUCCION}

La dieta por sí sola no puede explicar el desarrollo de la caries dental, debido a su origen multifactorial. Más aún, ella por sí misma es multifactorial. Qué y cómo se alimenta una persona son dos factores importantes para el desarrollo de caries dental, con cierta independencia entre uno y el otro $(1,2)$.

König propone que la asesoría dietética no es necesaria para el mantenimiento de la salud en general (3). Mientras, Zero concluye que, si bien la relación entre el consumo de azúcares y la caries dental no es tan marcada como en la época anterior a los fluoruros, la restricción del consumo de azúcares aún tiene un rol importante en la prevención de la caries dental $(4,10)$.

Los hábitos dietéticos cambian de una sociedad a otra. Los nuevos estilos de vida han producido variaciones, de las tres comidas principales en familias comer poco, varias veces al día y a solas (grazingo snacking), aumentando el riesgo de caries dental (5).

Se dice que es imposible cambiar los hábitos dietéticos. Sin embargo, durante la evolución, los hábitos de los individuos y de las distintas poblaciones han cambiado gradualmente, $\mathrm{y}$ algunos estudios muestran que puede alcanzarse con programas dietéticos estructurados $(6,7)$.

La asesoría dietética, en la modificación de hábitos, debe ser una herramienta de enseñanza reforzada, de fácil manejo e interpretación (5) .Sus objetivos primarios son: 1) Proveer una dieta completa 2) Evitar enfermedades relacionadas con la dieta y 3) Prevenir la transmisión de enfermedades originadas en los alimentos (8)

Perssonet y Granathet. Encontraron que la asesoría dietética presenta una mejor eficiencia "preventiva" que la higiene oral y las tabletas de flúor masticables $(8,9)$.

Pero el desconocimiento de los alimentos que afectan la salud dental y la motivación que necesitan las personas para que puedan cambiar los alimentos dañinos son otros motivos que hacen necesaria la asesoría dietética $(7,9)$.
El mensaje "comer menos azúcares la menor cantidad de ocasiones posibles" necesita ser modificado (6), pues los cambios dietéticos forzados no son exitosos, a menos que los beneficios se manifiesten rápidamente $\mathrm{y}$ den una ventaja real. De otro modo son rápidamente abandonados.

La asesoría dietética debe darse en un contexto de prevención total, basado en los patrones de ingesta, necesidades específicas (Ejem. compatibilidad con la medicación, alergias alimenticias, etc.) y con la situación socioeconómica del paciente; adaptándose a los estilos de vida de cada individuo $(1,2,6,11,12)$.

Otra manera de mejorar los hábitos dietéticos es mediante la formulación de políticas públicas de salud oral. Los aspectos comunitarios permiten la posibilidad de políticas y legislación que limiten la disponibilidad de azúcares, como advertencias en los empaques y reglas para la venta de productos con contenido azucarado cerca de áreas escolares. Aunque, este tipo de programa dietético no es fácil de lograr por las consideraciones económicas, especialmente en los países en desarrollo (13).

En este contexto, la educación en salud oral para reducir el consumo de los azúcares es de urgencia $(1,13)$.

Este estudio pretende determinar si la asesoría dietética efectuada en niños de 3 a 13 años de edad atendidos en la Clínica Estomatológica Central de la Universidad Peruana Cayetano Heredia, en un decenio, tuvo efecto sobre la frecuencia de consumo de azúcares extrínsecos.

\section{MATERIALES Y MÉTODOS}

Se revisó la base de datos de la Clínica Dental, de la Facultad de Estomatología de la Universidad Peruana Cayetano Heredia, constituida por pacientes atendidos en las asignaturas clínicas a cargo el Departamento Académico de Estomatología del Niño y del Adolescente (DAENA).

La muestra estuvo conformada por 2865 historias clínicas de pacientes entre 3 y13 años de edad. Se consideraron las siguientes variables: 
Frecuencia diaria del consumo de azúcares extrínsecos (FCAE): Es el número de veces al día que el paciente consume alimentos que contienen azúcares extrínsecos. Se obtiene de un diario dietético.

Asesorías dietéticas recibidas: Consejo dietético que se entrega al apoderado del paciente con algunas recomendaciones sobre sus hábitos dietéticos, luego de realizar el análisis dietético.

Categorías: "No recibió ninguna asesoría dietética", "Recibió solo una asesoría dietética" y "Recibió dos asesorías dietéticas".

Covariables:

- Género: Determinada según las características externas (fenotípicas) de cada individuo. Puede ser femenino o masculino.

- Edad: Se mide en años cumplidos en el momento del registro del paciente en la Clínica Estomatológica Central. Pueden ser de 3 a 5, de 6 a 8, de 9 a 11 o de 12 a 13 años.

- Período de tiempo evaluado: Número de años analizados en conjunto. Ejem.: lustro, década, etc. Pueden ser el lustro 1994-1998 ó 1999-2003.

\section{RESULTADOS}

De la población, el 47,2\% eran de sexo masculino y el 52,8\% de sexo femenino. E1 29,0\% tenía de 3 a 5 años; el 45,9\%, de 6 a 8 años; el 20,9\%, de 9 a 11 años y el 4,3\%, de 12 a13 años. E1 52,2\% de los pacientes fueron atendidos durante el período 1994-1998 y el $47,8 \%$, entre 1999 y 2003.

El 13,1\% no recibió asesoría dietética, el 73\% recibió solo una asesoría y el 13,9\%, dos. Al comparar el número de asesorías dietéticas recibidas, según las covariables, no se encontraron diferencias según el sexo de los pacientes y el período de tiempo evaluado (Tabla 1). Sin embargo, el porcentaje de pacientes que no recibió asesoría dietética se incrementó conforme se incrementaba la edad, desde $10,6 \%$ entre niños de 3 a 5 años hasta el $21,3 \%$, entre niños de 11 a 12 años (Tabla 1).

La FCAE se incrementó progresivamente con el transcurso del tiempo, de un promedio de $2,91+1,16$ en la evaluación inicial, a 3,29 + 1,37 en la evaluación intermedia y a 3,33+1,39 en la evaluación final. La tabla 2 muestra la comparación de la FCAE inicial, intermedia y final según las covariables. Se encontró que durante la evaluación inicial, la FCAE fue mayor en el sexo masculino $(2,94)$, que en el femenino $(2,88)$, mientras que durante las evaluaciones intermedia y final, el sexo femenino tuvo una FCAE mayor que el sexo masculino $(3,32$ versus 3,25 y 3,38 versus 3,28 respectivamente). Según la edad, se encontró que los niños de 9 a 11 años presentaron la mayor FCAE en las evaluaciones inicial e intermedia $(3,05$ y 3,35 respectivamente), mientras que los niños de 6 a 8 años presentaron la mayor FCAE en la evaluación

Tabla 1. Asesorías dietéticas recibidas según covariables

\begin{tabular}{|c|c|c|c|c|c|c|}
\hline \multirow{3}{*}{ Covariables } & \multicolumn{6}{|c|}{ Número de asesorías dietéticas recibidas } \\
\hline & \multicolumn{2}{|c|}{ Ninguna } & \multicolumn{2}{|c|}{ Solo una } & \multicolumn{2}{|c|}{ os } \\
\hline & $\mathrm{n}$ & $\%$ & $\mathrm{n}$ & $\%$ & $\mathrm{n}$ & $\%$ \\
\hline \multicolumn{7}{|l|}{ Género } \\
\hline Masculino & 167 & 12,4 & 1000 & 74,0 & 184 & 13,6 \\
\hline Femenino & 208 & 13,7 & 1092 & 72,2 & 214 & 14,1 \\
\hline \multicolumn{7}{|l|}{ Edad en años } \\
\hline 3 a 5 & 88 & 10,6 & 619 & 74,6 & 123 & 14,8 \\
\hline 6 a 8 & 165 & 12,6 & 984 & 74,8 & 165 & 12,6 \\
\hline 9 a 11 & 96 & 16,0 & 405 & 67,6 & 98 & 16,4 \\
\hline 12 a 13 & 26 & 21,3 & 84 & 68,9 & 12 & 9,8 \\
\hline \multicolumn{7}{|l|}{ Lustro evaluado } \\
\hline 1994-1998 & 177 & 11,8 & 1113 & 74,4 & 206 & 13,8 \\
\hline 1999-2003 & 198 & 14,5 & 979 & 71,5 & 192 & 14,0 \\
\hline
\end{tabular}


Tabla 2. Frecuencia de consumo de azúcares extrínsecos según covariables

\begin{tabular}{|c|c|c|c|c|c|c|c|}
\hline \multirow{3}{*}{ Covariables } & \multirow{3}{*}{$\mathrm{n}$} & \multicolumn{6}{|c|}{ Frecuencia de consumo de azúcares extrínsecos } \\
\hline & & \multicolumn{2}{|c|}{ Eval. Inicial } & \multicolumn{2}{|c|}{ Eval. Intermedia } & \multicolumn{2}{|c|}{ Eval. Final } \\
\hline & & Media & D.E. & Media & D.E. & Media & D.E. \\
\hline \multicolumn{8}{|l|}{ Género } \\
\hline Masculino & 1351 & 2,94 & 1,16 & 3,25 & 1,35 & 3,28 & 1,38 \\
\hline Femenino & 1514 & 2,88 & 1,16 & 3,32 & 1,38 & 3,38 & 1,40 \\
\hline \multicolumn{8}{|l|}{ Edad en años } \\
\hline 3 a 5 & 830 & 2,84 & 1,15 & 3,26 & 1,39 & 3,30 & 1,43 \\
\hline 6 a 8 & 1314 & 2,89 & 1,15 & 3.31 & 1,37 & 3,39 & 1,37 \\
\hline 9 a 11 & 599 & 3,05 & 1,14 & 3,35 & 1,36 & 3,31 & 1,40 \\
\hline 12 a 13 & 122 & 2,90 & 1,38 & 3,03 & 1,22 & 3,10 & 1,30 \\
\hline \multicolumn{8}{|l|}{ Lustro evaluado } \\
\hline 1994-1998 & 1496 & 2,89 & 1,17 & 3,23 & 1,37 & 3,26 & 1,39 \\
\hline $1999-2003$ & 1369 & 2,93 & 1,15 & 3,36 & 1,36 & 3,41 & 1,38 \\
\hline
\end{tabular}

final $(3,39)$. Según el período de tiempo evaluado se encontró una mayor FCAE durante el lustro 19992003 que durante 1994-1998 en las tres evaluaciones realizadas.

Al comparar la FCAE inicial, intermedia y final según el número de asesorías dietéticas recibidas,

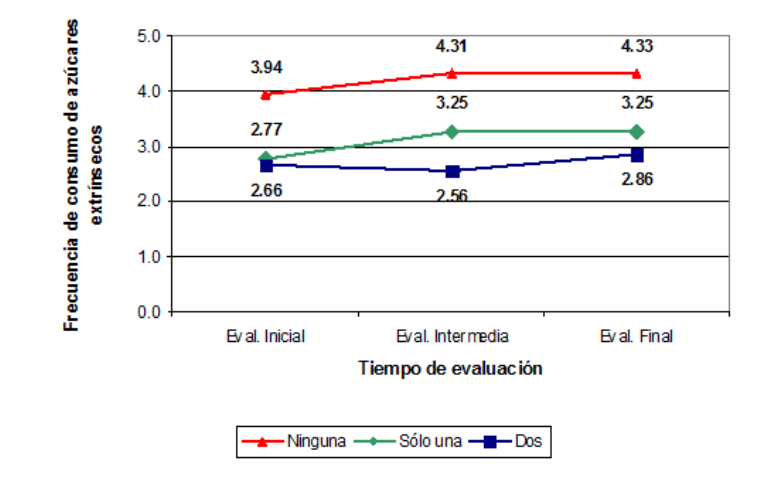

Gráfico 1. Frecuencia de consumo de azúcares extrínsecos según las asesorías dietéticas recibidas

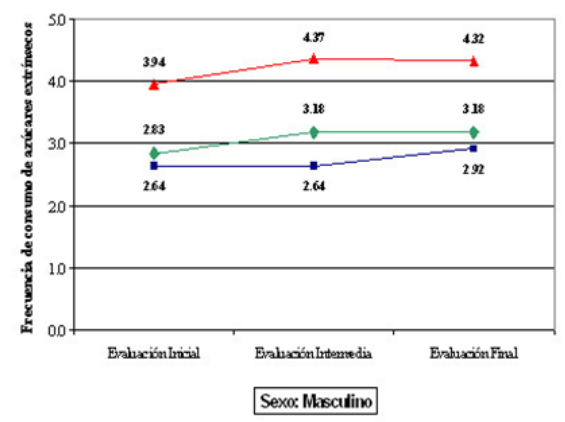

\-Nhgura $\rightarrow$-Silowa $\rightarrow$-Antas

Gráfico 2. Frecuencia de consumo de azúcares extrínsecos de acuerdo a las asesorías dietéticas recibidas, segúny Género. se encontró que el grupo que recibió dos asesorías dietéticas tuvo la menor FCAE mientras que el grupo que no recibió asesorías tuvo la mayor FCAE durante las tres evaluaciones realizadas (Tabla 3, Gráfico 1).Además, el incremento en la FCAE entre las evaluaciones inicial y final fue mayor en el grupo que recibió solo una asesoría dietética $(0,48)$ y menor en el grupo que recibió dos asesorías $(0,20)$.

Finalmente, la FCAE inicial, intermedia y final de acuerdo al número de asesorías dietéticas recibidas según covariables se muestra en la Tabla 4 y en los gráficos 2, 3 y 4. Según el sexo, en el grupo con dos asesorías dietéticas, se encontró que el sexo femenino disminuyó la FCAE entre las evaluaciones inicial e intermedia (de 2,68 a 2,49) mientras el sexo masculino se mantuvo (2,64 en ambas evaluaciones).Asimismo, en el grupo que no recibió asesoría dietética, el sexo femenino aumentó la FCAE entre las evaluaciones intermedia y final (de 4,27 a 4,34), mientras el sexo

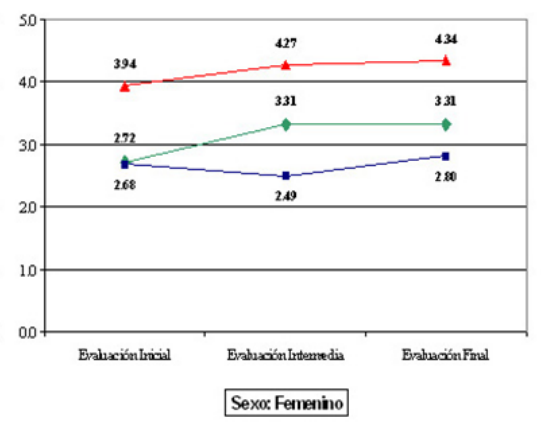

Sexo. Femenino 
Tabla 3. Frecuencia de consumo de azúcares extrínsecos según las asesorías dietéticas recibidas

\begin{tabular}{lccccccc}
\hline $\begin{array}{l}\text { Número de asesorías } \\
\text { dietéticas recibidas }\end{array}$ & $\mathrm{n}$ & \multicolumn{5}{c}{ Frecuencia de consumo de azúcares extrínsecos } \\
& \multicolumn{5}{c}{ Eval. Inicial } & Eval. Intermedia & Eval. Final \\
\hline \multirow{3}{*}{ Ninguna } & & Media & D.E. & Media & D.E. & Media & D.E. \\
Solo una & 375 & 3,94 & 1,34 & 4,31 & 1,22 & 4,33 & 1,19 \\
Dos & 2092 & 2,77 & 1,05 & 3,25 & 1,34 & 3,25 & 1,34 \\
Total & 398 & 2,66 & 1,02 & 2,56 & 1,02 & 2,86 & 1,37 \\
\hline
\end{tabular}

masculino disminuyó la FCAE (de 4,37 a 4,32) (Tabla 3, Gráfico 2). Según la edad, se encontró el mismo aumento en la FCAE entre las evaluaciones inicial y final para los tres grupos conformados según el número de asesorías dietéticas (Tabla 4), Gráfico 3, sin embargo el incremento fue menor en los grupos de mayor edad (9 a 11 y 12 a 13 años, Tabla 4, Gráfico 3. Según el periodo de tiempo evaluado, se encontró que durante el lustro 1994-1998 el grupo que no recibió asesoría dietética disminuyó su FCAE entre las evaluaciones intermedia y final (de 4,41 a 4,36), mientras que en el lustro 1999-2003 la FCAE aumentó (de 4,23 a 4,30).Asimismo, el descenso en el grupo con dos asesorías dietéticas entre las evaluaciones inicial e intermedia fue mayor en el lustro 1994-1998 que en 1999-2003. (Tabla 4, Gráfico 4).

\section{DISCUSIÓN}

Cuando se comparó el número de asesorías recibidas según el sexo no se encontró diferencias. Threfall evaluó los factores que influencian a los operadores para dar una asesoría dietética en la prevención de caries dental en niños y también encontró que el sexo no es importante cuando el dentista decide dar una asesoría dietética (14).

La disminución del número de pacientes que recibieron asesoría dietética conforme aumentaba la edad llevó a preguntarnos: ¿De qué manera se relaciona la edad del paciente con la asesoría dietética?

Para Threfall, la edad sí influye la entrega de la asesoría, de tal modo que los niños mayores deben ser incluidos en el proceso (14). En otro estudio en niños de 13 años de edad, donde todos los dientes permanentes estaban totalmente erupcionados, Hegde sostiene que durante esta edad existe un considerable riesgo y actividad de caries dental; por ello, la asesoría dietética es necesaria para prevenir la enfermedad (15). No debería ser difícil incluir a niños mayores en programas de asesoría dietética, más aún, si se tiene en cuenta que los hábitos dietéticos y de higiene se establecen en edades tempranas (16).

¿Qué factores afectan la entrega de la asesoría dietética? Al parecer, el factor más importante es el nivel de caries que presentan los pacientes. Así,

Tabla 4. Frecuencia de consumo de azúcares extrínsecos de acuerdo a las asesorías dietéticas recibidas, según covariables

\begin{tabular}{|c|c|c|c|c|c|c|c|c|c|c|c|c|c|c|c|c|c|c|}
\hline \multirow{4}{*}{ Covariables } & \multicolumn{18}{|c|}{ FCAE de acuerdo al número de asesorías dietéticas recibidas } \\
\hline & \multicolumn{6}{|c|}{ Evaluación Inicial } & \multicolumn{6}{|c|}{ Evaluación Intermedia } & \multicolumn{4}{|c|}{ Evaluación Final } & & \\
\hline & \multicolumn{2}{|c|}{ Ninguna } & \multicolumn{2}{|c|}{ Solo una } & \multicolumn{2}{|c|}{ Dos } & \multicolumn{2}{|c|}{ Ninguna } & \multicolumn{2}{|c|}{ Solo una } & \multicolumn{2}{|c|}{ Dos } & \multicolumn{2}{|c|}{ Ninguna } & \multicolumn{2}{|c|}{ Solo una } & \multicolumn{2}{|c|}{ Dos } \\
\hline & Media & D.E. & Media & D.E. & Media & D.E. & Media & D.E. & Media & D.E. & Media & D.E. & Media & D.E. & Media & D.E. & Media & D.E. \\
\hline \multicolumn{19}{|l|}{ Género } \\
\hline Masculino & 3,94 & 1,41 & 2,83 & 1,04 & 2,64 & 1,07 & 4,37 & 1,21 & 3,18 & 1,32 & 2,64 & 1,02 & 4,32 & 1,26 & 3,18 & 1,32 & 2,92 & 1,35 \\
\hline Femenino & 3,94 & 1,29 & 2,72 & 1,05 & 2,68 & 0,98 & 4,27 & 1,23 & 3,31 & 1,36 & 2,49 & 1,01 & 4,34 & 1,13 & 3,31 & 1,36 & 2,80 & 1,38 \\
\hline \multicolumn{19}{|l|}{ Edad en años } \\
\hline 3 a 5 & 3,98 & 1,20 & 2,74 & 1,07 & 2,55 & 1,03 & 4,41 & 1,12 & 3,25 & 1,37 & 2,47 & 1,09 & 4,50 & 0,97 & 3,24 & 1,40 & 2,78 & 1,41 \\
\hline 6 a 8 & 3,74 & 1,48 & 2,78 & 1,05 & 2,69 & 0,97 & 4,30 & 1,25 & 3,26 & 1,35 & 2,58 & 0,99 & 4,23 & 1,27 & 3,30 & 1,33 & 3,05 & 1,37 \\
\hline 9 a 11 & 4,23 & 1,09 & 2,83 & 0,98 & 2,76 & 1,09 & 4,35 & 1,19 & 3,29 & 1,33 & 2,61 & 0,99 & 4,44 & 1,00 & 3,20 & 1,35 & 2,63 & 1,30 \\
\hline 12 a 13 & 4,00 & 1,60 & 2,60 & 1,16 & 2,67 & 1,16 & 3,92 & 1,47 & 2,81 & 1,06 & 2,67 & 0,78 & 4,00 & 1,74 & 2,86 & 1,02 & 2,83 & 1,12 \\
\hline \multicolumn{19}{|l|}{ Lustro evaluado } \\
\hline 1994-1998 & 3,98 & 1,39 & 2,78 & 1,05 & 2,60 & 1,05 & 4,41 & 1,18 & 3,16 & 1,34 & 2,58 & 1,06 & 4,36 & 1,24 & 3,16 & 1,33 & 2,84 & 1,36 \\
\hline 1999-2003 & 3,90 & 1,30 & 2,77 & 1,04 & 2,73 & 0,98 & 4,23 & 1,25 & 3,35 & 1,34 & 2,54 & 0,97 & 4,30 & 1,14 & 3,34 & 1,35 & 2,88 & 1,37 \\
\hline
\end{tabular}



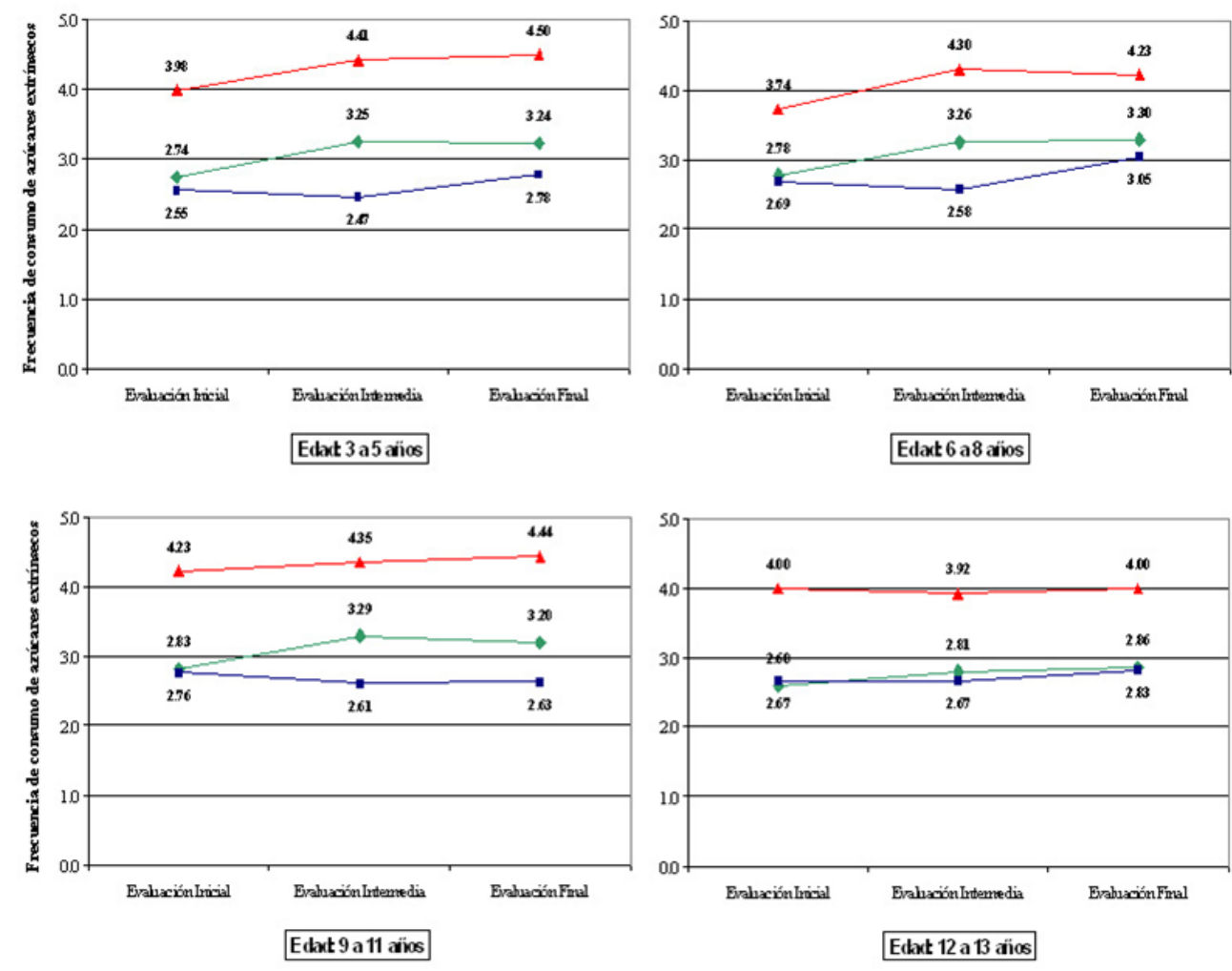

$\rightarrow$ Norguma $\rightarrow$ Solouma $\rightarrow-$ Anbas

Gráfico 3. Frecuencia de consumo de azúcares extrínsecos de acuerdo a las asesorías dietéticas recibidas, según edad.

los niños con niveles altos de caries dental son más "indicados" para ser interrogados sobre la dieta e higiene oral (14).

La percepción del operador sobre los padres de familia, especialmente sobre sus creencias y actitudes, también es crucial al momento de dar una asesoría. Si el operador percibe el interés de los padres, se siente más motivado para dar una asesoría más detallada. Muchos dentistas admiten su desilusión y pérdida de motivación al dar la asesoría dietética cuando no se sienten escuchados o por la falta de atención de los pacientes (14).

El tiempo es otro factor importante. La mayoría de los operadores cree que la educación es la llave de la prevención; sin embargo, tienen grandes restricciones de tiempo relacionadas con deficiencias en la estructura salarial (costo vs. uso eficiente de tiempo $(14,17)$.

También, nuestro estudio encontró un aumento progresivo de la FCAE en las diferentes evaluaciones de la dieta, a pesar de la asesoría dietética. En otros términos, es evidente que la asesoría dietética no consiguió su objetivo (reducir la FCAE). La pregunta surge por sí sola: ¿por qué?

La literatura nos dice que aunque hay poca evidencia de efectividad en la asesoría dietética, la existente refleja un efecto débil y transitorio. La mayoría de los estudios muestra un cambio en los conocimientos de los pacientes, pero no en sus hábitos . Esto explicaría la disminución de la FCAE en el grupo con dos asesorías entre las evaluaciones inicial e intermedia y su reincidencia entre las evaluaciones intermedia y final.

Según la Federación Dental Internacional (FDI) es importante una apropiada diferenciación individual cuando se da una asesoría dietética, dado que no todas las recomendaciones son igualmente aplicables en todos los países y poblaciones. También debemos de tener en cuenta que el impacto de una asesoría en la salud oral de los pacientes pediátricos puede estar influenciado no solo por el contenido sino también por cómo y a quién está dirigida, ya que por lo general la asesoría es dada de manera general sin ninguna individualización (14). 

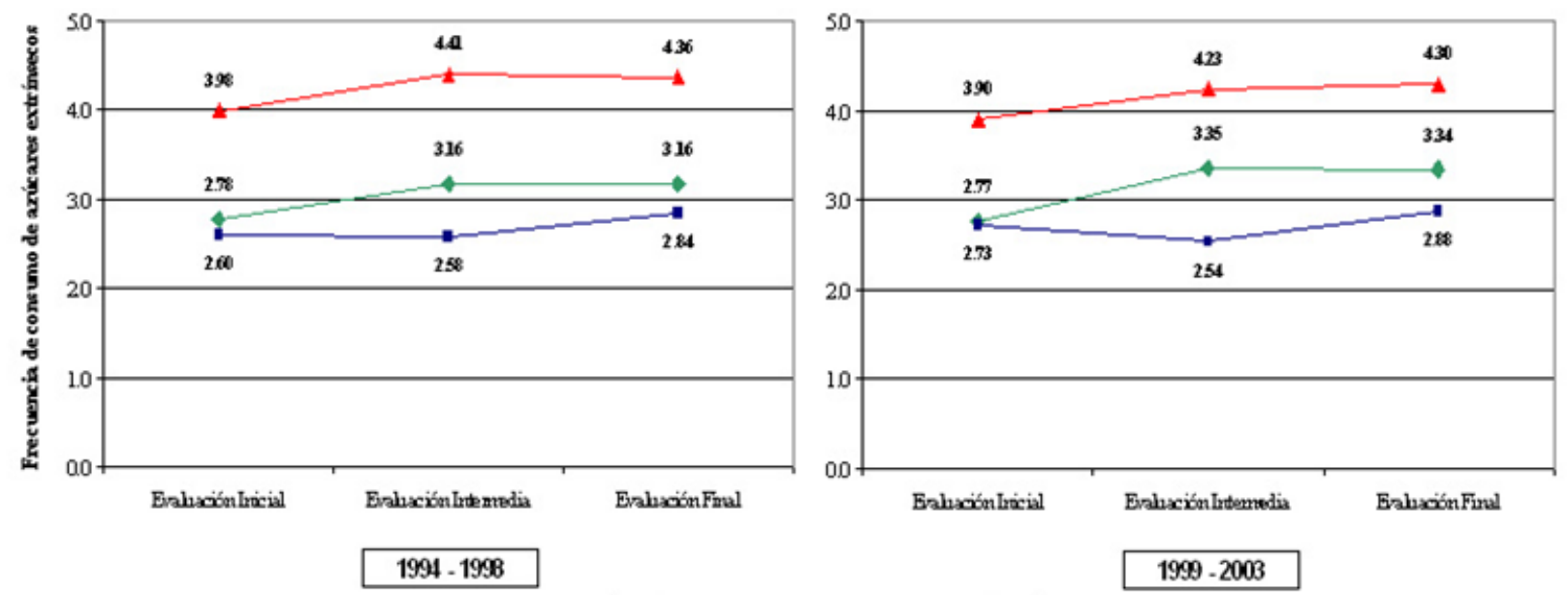

$\rightarrow$ Kingura $\rightarrow$ Sólouna $\rightarrow$ Antas

Gráfico 4. Frecuencia de consumo de azúcares extrínsecos de acuerdo a las asesorías dietéticas recibidas, según lustro evaluado.

La mayoría de los dentistas realizan una asesoría verbal, una especie de charla educativa, y en pocos casos usan materiales adicionales. Por lo tanto, existe un vacío creativo en la manera de dar la asesoría dietética. Muchos estudios han demostrado que el uso de métodos específicos para niños pequeños, como juegos, ayuda a reforzar los mensajes de salud oral (14). Ayudas visuales como la devolución de los diarios dietéticos a los niños, donde los alimentos azucarados son encerrados con círculos rojos, permiten identificar los alimentos cariógenos y no cariógenos (15). Adicionalmente, se debería contar con material extra que los padres puedan llevar a casa (14).

Otro problema común es que los odontólogos se ven provocados a delegar la consejería dietética y la instrucción de higiene oral a los higienistas dentales, olvidando que es el dentista quien debe proveer el mensaje preventivo, y el higienista reforzar los conceptos en la práctica junto al paciente o dar ejemplos prácticos (14).

Tampoco olvidemos que la asesoría dietética casi siempre está enfocada en mensajes negativos (Ejem.: bebidas con alto contenido de azúcares dañinas para los dientes). Moynihan afirma que los mensajes positivos son más fáciles de seguir. Por lo tanto, la promoción de alimentos conocidos como protectores de los dientes es una parte importante de la asesoría dietética que permitirá la entrega de mensajes positivos (18).
Asimismo encontramos diferencias en el consumo de azúcares extrínsecos entre niños y niñas en las distintas evaluaciones. Estos hallazgos concuerdan con Aracella, quien explica que estas diferencias se deben a los distintos patrones dietéticos entre hombres y mujeres (19). Kiwanuka tuvo resultados similares, cuando evaluó la FCAE de escolares en Uganda, los niveles promedio de la FCAE fueron mayores en las niñas que en los niños (20).

Para Bozal y Romero, quienes evaluaron la dieta de 37 niños entre 6 y 14 años de edad, no existe relación entre la FCAE y el sexo (21). De modo semejante, Hegde, al comparar los valores promedio entre ambos sexos con respecto de la FCAE, mostró que el consumo fue ligeramente mayor en el sexo masculino, pero no se observaron diferencias significativas (15). De modo similar, Sarmadi menciona que los niños tienen mayor inflamación gingival que las niñas, consecuencia del consumo de FCAE y una higiene deficiente (10).

En cuanto a las diferencias entre la edad y la FCAE, encontramos que los niños más grandes presentan mayores niveles promedio de aumento de la FCAE que los menores. De modo similar, Kiwanuka encontró que los niveles promedio de la FCAE fueron mayores en alumnos de los mayores grados de escolaridad que en los de grados menores (20). Probablemente es porque los niños mayores son más independientes de sus padres, de quienes reciben propinas, lo que facilita la obtención de golosinas; mientras los más pequeños solo consumen los alimentos que sus padres les proporcionan (22). 
En el segundo lustro (1999-2003) se encontraron valores mayores de la FCAE que en el primero (19941998). Un hecho similar fue observado por RuggGunn, quien analizó los cambios de consumo de azúcares en adolescentes en Inglaterra y encontró que la FCAE había aumentado durante un periodo de 20 años (1980 - 2000) (9). Esto podría explicarse, según König, porque la FCAE está directamente relacionada con la libre disponibilidad de los azúcares en el mercado para su consumo (3). De acuerdo al análisis de los mercados mundiales sobre las perspectivas alimentarias de la Organización de las Naciones Unidas para la Agricultura y la Alimentación (FAO), actualmente existe una gran cantidad de excedentes en la producción de azúcar que genera un descenso constante de su precio hasta alcanzar niveles sin precedentes en los últimos años, permitiendo su mayor consumo(23).

Acerca de la diferencias entre la FCAE y el número de asesorías dietéticas recibidas, encontramos que el grupo que recibió dos asesorías dietéticas tuvo la menor FCAE mientras el grupo que no recibió asesoría tuvo la mayor FCAE durante las tres evaluaciones realizadas. Además, el incremento en la FCAE entre las evaluaciones inicial y final fue mayor en el grupo que recibió solo una asesoría dietética (0.48) y menor en el grupo que recibió dos asesorías (0.20). Esto revelaría que existen mejores resultados en los pacientes cuando reciben más de una asesoría dietética. Hasta el momento no existe literatura al respecto que fundamente o refute estos resultados.

\section{CORRESPONDENCIA}

\author{
Abraham Meneses López \\ Av. Honorio Delgado 430, Lima 31 - Perú \\ Teléfono: $381-2584$ \\ e-mail:ameneses@metacrawler.com
}

\section{REFERENCIAS BIBLIOGRÁFICAS}

1. Birked D. Behavioural aspects of dietary habits and dental caries. Caries Res 1990;24(1):27-35

2. Nunn ME, Braunstein NS, Krall Kaye EA, et al. Healthy eating index is a predictor of early childhood caries. $\mathrm{J}$ Dent Res 2009;88(4):361-6.

3. König KG. Changes in the prevalence of dental caries: how much can be attributed to changes in diet? Caries Res 1992;24():16-8
4. Zero DT. Sugars - the arch criminal? Caries Res 2004;38:277-85.

5. Van Loveren C, Duggal MS. Experts'opinions on the role of diet in caries prevention. Caries Res 2004;38(19:1623

6. Johansson I. Diet counselling and behaviour change. Caries Res 1993;27(1):47-9

7. Rugg-Gunn AJ, Fletcher ES, Matthews JN, et al. Changes in consumption of sugars by English adolescents over 20 years. Public Health Nutr 2007;10(4):354-63.

8. Marthaler TM. Changes in the prevalence of dental caries: how much can be attributed to changes in diet? Caries Res 1990;24(1):3-15.

9. Bibby BG. Food and the teeth. New York (LA): Vantage Press; 1990. p. 71-7

10.Sarmadi R, Gahnberg L, Gabre P. Clinicians' preventive strategies for children and adolescents identified as at high risk of developing caries. Int $\mathrm{J}$ Paediatr Dent 2011;21(3):167-74.

11.Edmonson EMS. Food composition and food cariogenicity factors affecting the cariogenic potential of foods. Caries Res 1990;24(1):60-71

12.Marshall TA. Chairside diet assessment of caries risk. J Am Dent Assoc 2009;140(6):670-4.

13.Sanders TAB. Diet and general health: dietary counselling. Caries Res. 2004;38(1):3-8.

14.Threlfall AG, Hunt CM, Milsom KM, Tickle M, Blinkhorn AS. Exploring factors that influence general dental practitioners when providing advice to help prevent caries in children. Br Dent J 2007 Feb 24;2004(4):E10.

15.Hegde PP, Ashok Kumar BR, Ankola A. Sugar consumption pattern of 13-year-old school children in Belgaum city, Karnataka. J Indian Soc Pedod Prev Dent. 2005;23:165-7.

16.Garbin CAS, Arcieri RM, Ferreira NF, Luvizuto ER, Alle CF. Assessment of the diet of 0- to 6-year-old children in municipal schools in a Brazilian city. $\mathrm{J}$ Indian Soc Pedod Prev Dent. 2005;23(3):119-23.

17.Harris R, Bridgman C. Introducing care pathway commissioning to primary dental care: the concept. $\mathrm{Br}$ Dent J. 2010;209(5):233-9.

18.Moynihan PJ. Food and dietary factors that prevent dental caries. Quintessence Int. 2007 Apr;38(4):320-4.

19.Aracella D, Ottolenghi L, Polimeni A, Leclerq C. The relationship between frequency of carbohydrates intake and dental caries: a cross-sectional study in italian teenagers. J Public Health Nutrition 2002;5(4):533-60.

20.Kiwanuka SN, Ástrøm AN, Trovik TA. Sugar snack cosumption in Ugandan schoolchildren: validity and reliability of a food frequency questionnaire. Community Dent Oral Epidemiol. 2006;34:372-80.

21.Bozal CB, Romero SB. Estudio sobre hábitos dietéticos 
en niños. Boletín de la Asociación Argentina de Odontología para Niños. 2001 Mar-Jun;30(1):3-10.

22. Roberts BP, Blinkhorn AS, Duxbury JT. The power of children over adults when obtaining sweet snacks. Int J
Paediatr Dent. 2003 Mar;13(2):76-84.

23. Organización de las Naciones Unidas para la Agricultura y la Alimentación (FAO). Perspectivas alimentarias: análisis de los mercados mundiales. Roma: FAO; 2007.

Recibido : 19-07-2012

Aceptado: 10-10-2012 